\title{
Efecto de una consulta nutricional protocolizada sobre el estado nutricional y hábitos alimentarios de adultos con sobrepeso y obesidad
}

\section{Effect of a structured nutritional consultation on weight loss and eating habits in overweight and obese adults}

\section{RESUMEN}

El sobrepeso y la obesidad afectan a un $67 \%$ de la población chilena. Se han realizado diferentes estrategias para revertir esta situación, siendo una de ellas es la realización de la consulta nutricional. No obstante, hasta la fecha ésta no se ha protocolizado y se desconoce si esto podría generar un efecto positivo en la reducción del exceso de peso corporal. El objetivo de este estudio fue evaluar el efecto de una consulta nutricional protocolizada (CNP) sobre el estado nutricional y los hábitos alimentarios en adultos con sobrepeso y obesidad en un centro de salud familiar. Se realizó un estudio de pre-post intervención en 30 participantes durante 3 meses. Se evaluó por antropometría, la variación del estado nutricional y por método dietario, la modificación de los hábitos alimentarios. La CNP produjo una disminución significativa en las variables antropométricas estudiadas (peso corporal, IMC, circunferencia de cintura y porcentaje de grasa corporal). Existieron mejorías en los hábitos alimentarios estudiados. Un 92\% de los participantes logró incorporar una colación de media mañana y una porción de lácteos descremados diario. A pesar de los efectos positivos obtenidos por la CNP es necesario que se corroboren estos beneficios en estudios de mayor duración, con un grupo representativo de participantes.

Palabras claves: consulta nutricional protocolizada, estado nutricional, hábitos alimentarios.

\section{ABSTRACT}

Overweight and obesity affect $67 \%$ of population in Chile. Different strategies have been developed to reverse and improve this situation, nutritional consultation is one such strategy. However, consultation has not been structured and it is unknown whether this procedure has a positive effect for weight loss. The objective of this study was to assess the effectiveness of a structured nutritional consultation on weight loss and eating habits in overweight and obese adults in a family health center. A pre-post intervention study was performed with 30 participants over 3 months. Anthropometric measures (weight, body mass index (BMI) and waist circumference (WC) and \% body fat), dietary intake and eating habits were the main outcomes of the
Fanny Petermann Rocha'; Eliana Durán Fernández²; Ana María Labraña Torres ${ }^{2}$ y Carlos Celis-Morales ${ }^{3}$

1. Programa de Magister en Nutrición Humana, Departamento de Nutrición y Dietética, Universidad de Concepción, Edmundo Larenas 215, Concepción 4030000, Chile. 2. Departamento de Nutrición y Dietética, Universidad de Concepción, Edmundo Larenas 215, Concepción 4030000, Chile. 3. BHF Glasgow Cardiovascular Research Centre, Institute of Cardiovascular and Medical Science, University of Glasgow, Glasgow, G12 8TA, United Kingdom.

Dirigir correspondencia a: Fanny Petermann Rocha, Nutricionista, MSC en Nutrición Humana, Programa de Magister en Nutrición Humana, Universidad de Concepción, Edmundo Larenas 215, Concepción 4030000, Chile. Teléfono: 56-41-2470424. E-mail: fannypetermann@udec.cl

Este trabajo fue recibido el 28 de abril de 2017. Aceptado con modificaciones: 05 de junio de 2017. Aceptado para ser publicado: 20 de agosto de 2017.

study. A structured nutritional consultation was significantly associated with decreased weight, BMI, WC and \% body fat. There were improvements in dietary intake habits. A 92\% of the participants incorporated a snack mid-morning and a daily serving of low-fat dairy. Despite benefits observed after a structured nutritional consultation, larger studies with longer duration are warranted.

Keywords: Structured nutritional consultation, body weight, eating habits. 


\section{INTRODUCCIÓN}

La obesidad se define como una acumulación anormal o excesiva de grasa corporal perjudicial para la salud'. Según cifras de la Organización Mundial de la Salud (OMS), en el año 2014 un 39\% de la población mayor de 18 años, a nivel mundial, presentaba sobrepeso y un $13 \%$ obesidad $^{2}$. Chile no queda ajeno a estas cifras. La última Encuesta Nacional de Salud realizada en el año 2009-2010 evidenció que un $67 \%$ de la población mayor de 18 años presenta exceso de peso según índice de masa corporal $\left(\mathrm{IMC} \geq 25 \mathrm{~kg} / \mathrm{m}^{2}\right)^{3}$. Esta cifra nacional es preocupante ya que más de 4 millones de muertes en el mundo están asociadas a obesidad, siendo las enfermedades cardiovasculares la principal causa de muerte asociada a un alto IMC'.

En Chile, los servicios de la atención primaria de salud podrían ser un espacio óptimo para implementar estrategias de intervención orientadas a disminuir la obesidad a nivel nacional, ya que, cerca del $80 \%$ de la población chilena accede a estos servicios ${ }^{4}$. El Ministerio de Salud de Chile (MINSAL), ha creado distintos programas de atención primaria para combatir diversas patologías y factores de riesgo, entre los cuales se encuentra la consulta nutricional inmersa en los distintos programas de salud ${ }^{5-7}$. La consulta nutricional se define como la atención dietética o dietoterapéutica proporcionada al individuo que requiere de alguna adecuación fisiológica o que presente alguna alteración de tipo fisiopatológica o nutricional, para modificar o reforzar su alimentación y nutrición, otorgando una atención adecuada acorde a la situación de salud de cada persona. Esta atención ofrece un gran potencial a todo nivel para la promoción de hábitos alimentarios saludables, cambios de conducta alimentaria y en consecuencia mejoras en el perfil antropométrico ${ }^{8}$.

Estudios muestran que la intervención periódica por nutricionistas, quincenal o mensual, posee efectos positivos principalmente en la disminución del peso corporal, obesidad central y mejorías en los hábitos alimentarios ${ }^{9-11}$. Sin embargo, la consulta nutricional no ha logrado disminuir los niveles de obesidad en la población reflejado en las altas cifras de sobrepeso y obesidad en el país ${ }^{3,12}$. Entre los factores que podrían explicar esta situación se encuentran: un tiempo reducido para la atención, siendo el mínimo nacional de 20 minutos, y la falta de una protocolización que permitiría hacer más efectivo el uso del tiempo en la consulta nutricional. Outomuro y cols., han descrito que tiempos cortos de atención es uno de los principales factores que impiden una adecuada atención en salud ${ }^{13}$. Esta situación no es diferente a lo que ocurre en la consulta nutricional en Chile, la que en la actualidad destina en algunos centros 30 minutos para la primera consulta nutricional y 15 minutos para los controles, tiempo en el que también se deben completar los formularios administrativos. A su vez la consulta nutricional actual no cuenta con un protocolo estandarizado a nivel nacional que permita optimizar el uso del tiempo destinado a esta consulta y así también sus beneficios.

Optimizar la consulta nutricional a través de su protocolización y con un tiempo de atención de 30 minutos destinado sólo a actividades técnicas, podría ser una potencial solución a este problema. Frente a lo anterior, el objetivo de este estudio fue evaluar el efecto de una consulta nutricional protocolizada sobre el estado nutricional y los hábitos alimentarios en adultos con sobrepeso y obesidad en un Centro de Salud Familiar.

\section{MATERIALES Y MÉTODOS}

Se realizó un estudio cuasi-experimental, el cual incluyó solamente a un grupo de intervención, el cual fue evaluado antes y posterior a una intervención de tres meses de duración. Durante estos tres meses (desde septiembre a diciembre del año 2015) cada participante asistió a la consulta nutricional protocolizada (CNP) en tres ocasiones (una vez al mes) en la que se realizaron las mediciones antropométricas y de hábitos alimentarios por la misma nutricionista a cargo de la investigación y externa al centro de salud, quien fue capacitada para la estandarización del protocolo de evaluación de CNP. Los datos generales recolectados durante estas tres consultas nutricionales protocolizadas correspondieron a sexo, edad, estado civil, nivel educacional y actividad laboral.

Selección de muestra y creación de protocolo de consulta nutricional protocolizada (CNP)

Se trabajó con un muestreo por conveniencia el que alcanzó a 30 participantes. El reclutamiento se realizó a través del Servicio de Orientación Médico Estadístico (SOME). Entre los criterios de inclusión se consideraron tanto a hombres como a mujeres entre 18 a 60 años de edad que presentaron diagnóstico de sobrepeso u obesidad a través del IMC kg/m². Se excluyó a aquellas personas que presentaron diagnóstico de diabetes mellitus tipo 2 y que hubiesen asistido en más de tres ocasiones a una consulta nutricional previa.

Se elaboró un protocolo para la CNP el que fue validado preliminarmente por juicio de expertos y por una muestra preliminar de 4 personas. Se aplicó el protocolo en cada una de las sesiones que asistieron los participantes con una duración total de 30 minutos por sesión desglosado según las acciones a realizar. Para esta construcción se realizó una búsqueda bibliográfica, búsqueda en documentos oficiales y consultas al MINSAL de Chile, para identificar los componentes actuales de una consulta nutricional no protocolizada y los que debería poseer una CNP y sus tiempos en minutos. Las acciones y sus tiempos que se consideraron se observan en la Tabla 1.

\section{Antropometría}

Se midió el peso corporal en cada consulta a través de una balanza marca Seca (modelo 700) con cartabón incorporado y con una precisión de 0,1 kg. La talla se midió en centímetros $(\mathrm{cm})$ a través del tallímetro de la misma balanza con una precisión de $0,1 \mathrm{~cm}$. Con estos dos datos se calculó el IMC en $\mathrm{kg} / \mathrm{m}^{2}$, cuyos valores se clasificaron acorde al reporte del año 2000 de la $\mathrm{OMS}^{14}$. 
Tabla 1

Acciones y tiempos de la consulta nutricional protocolizada

\begin{tabular}{|c|c|c|}
\hline Acciones & $\begin{array}{l}\text { Tiempo en } \\
\text { minutos }\end{array}$ & Detalle de la acción \\
\hline $\begin{array}{l}\text { Saludo y } \\
\text { presentación }\end{array}$ & 0,5 & $\begin{array}{l}\text { El profesional saludará al usuario y acompañante. Indicará su nombre y su disposición a } \\
\text { resolver sus necesidades. }\end{array}$ \\
\hline $\begin{array}{l}\text { Motivo } \\
\text { consulta }\end{array}$ & 0,5 & $\begin{array}{l}\text { Se consultará al usuario motivo de la consulta. } \\
\text { ¿En qué puedo ayudarlo? ¿Por qué ha venido? ¿Quién lo derivó? }\end{array}$ \\
\hline $\begin{array}{l}\text { Anamnesis } \\
\text { Alimentaria }\end{array}$ & 5,5 & $\begin{array}{l}\text { Se preguntará si ha realizado una "dieta" o plan de alimentación anterior. De ser así los resultados } \\
\text { obtenidos y quién lo recomendó. } \\
\text { Se realizará encuesta alimentaria por interrogatorio cuyo propósito será identificar estructura global } \\
\text { de la alimentación y hábitos alimentarios. } \\
\text { Se aplicarán } 3 \text { encuestas alimentarias por interrogatorio: } \\
\text { Anamnesis alimentaria, Recordatorio de } 24 \text { horas y encuesta de tendencia de consumo } \\
\text { semanal modificada. } \\
\text { • Anamnesis alimentaria. } \\
\text { Se preguntará por: Apetito, ansiedad, lugar dónde consume los alimentos, con quién realiza las } \\
\text { comidas, No de comidas que realiza al día, horas de ayuna, quién prepara los alimentos, velocidad } \\
\text { al comer, preferencias alimentarias, intolerancias alimentarias y finalmente ingesta de líquidos } \\
\text { totales desglosado en consumo de agua y otras infusiones. } \\
\text { - Encuesta recordatorio de } 24 \text { horas } \\
\text { Se aplicará una en cada consulta para determinar horarios, tiempos de comidas y las preparaciones } \\
\text { consumidas detalladas por ingredientes. } \\
\text { - Encuesta de tendencia de consumo semanal modificada } \\
\text { Se aplicará esta encuesta durante cada consulta nutricional. Se preguntará sobre el consumo } \\
\text { semanal o quincenal de: } \\
\text { Pan, arroz, fideos, papas, frutas, verduras, lácteos, pescado, legumbres, carnes rojas, pollo, } \\
\text { vienesas, embutidos, aceite, palta, bebida, dulces y sal. }\end{array}$ \\
\hline $\begin{array}{l}\text { Antropometría } \\
\text { y examen } \\
\text { físico }\end{array}$ & 3 & $\begin{array}{l}\text { Se realizará la medición de: peso corporal, talla, circunferencia de cintura y medición de } \\
4 \text { pliegues cutáneos (bicipital, tricipital, subescapular y suprailíaco). Junto a ellos, se realizará } \\
\text { examen físico para la búsqueda de signos asociados a alguna patología como edemas, } \\
\text { hirsutismo, acantosis nigricans, entre otros. }\end{array}$ \\
\hline $\begin{array}{l}\text { Cálculo } \\
\text { requerimientos } \\
\text { nutricionales }\end{array}$ & 0,5 & $\begin{array}{l}\text { Para realizar el cálculo de requerimientos se utilizará peso ideal ajustado por talla y contextura } \\
\text { utilizando la norma establecida por el MINSAI (fórmula Knox). }\end{array}$ \\
\hline $\begin{array}{l}\text { Evaluación cuali } \\
\text { y cuantitativa } \\
\text { de la dieta }\end{array}$ & 0,5 & $\begin{array}{l}\text { Se calculará el indicador dietario para evaluar la suficiencia de la dieta. Se realizará al dividir el } \\
\text { aporte de energía (calorías) y nutrientes consumidos por aquellos requeridos y multiplicándolo } \\
\text { por } 100 \% \text {. Un valor menor al } 90 \% \text { es considerado insuficiente y mayor al } 110 \% \text { es considerado } \\
\text { excesivo. }\end{array}$ \\
\hline $\begin{array}{l}\text { Diagnóstico } \\
\text { nutricional } \\
\text { integrado }\end{array}$ & 1 & $\begin{array}{l}\text { Se interpretarán los valores de las distintas mediciones para realizar el diagnóstico nutricional } \\
\text { integrado. } \\
\text { - Según antropometría: Estado nutricional (sobrepeso u obesidad), \% de grasa corporal y } \\
\text { obesidad abdominal según circunferencia cintura. } \\
\text { - Método dietario: Grado de suficiencia de la alimentación y la participación relativa de la energía } \\
\text { según sustrato (valor energetico total, VET). }\end{array}$ \\
\hline
\end{tabular}




\begin{tabular}{|c|c|c|}
\hline $\begin{array}{l}\text { Educación } \\
\text { alimentaria } \\
\text { y entrega de } \\
\text { pauta } \\
\text { alimentaria }\end{array}$ & 12,5 & $\begin{array}{l}\text { Se realizará mediante conversación individual. En cada sesión se abordarán diferentes aristas: } \\
\text { - } \quad \text { Proceso por el cual se acumula la grasa (composición corporal) y/o en qué consiste su patología } \\
\text { - } \quad \text { de base (resistencia a la insulina, hipertensión o dislipidemia) } \\
\text { - } \quad \text { Requerimientos energéticos diarios. } \\
\text { - } \quad \text { Porciones de intercambio de alimentos. } \\
\text { - Opciones de preparaciones y guisos para consumo diario y/o semanal. } \\
\text { El plan de alimentación será ajustado según preferencias u otras variables. Se otorgará esta } \\
\text { información por escrito y se plantearán metas a cumplir hasta el próximo control. Se trabajará } \\
\text { en una meta por vez. }\end{array}$ \\
\hline $\begin{array}{l}\text { Derivaciones y } \\
\text { Citación }\end{array}$ & 0,5 & $\begin{array}{l}\text { Se derivará a otro profesional según corresponda y se citará para próxima consulta. La próxima } \\
\text { consulta nutricional protocolizada se realizará en un mes. }\end{array}$ \\
\hline Registro final & 0,5 & Se realizará registro en ficha electrónica y de papel según corresponda. \\
\hline
\end{tabular}

Antes de la evaluación antropométrica se le solicitó a cada participante que usara ropa ligera y se quitara los zapatos. La circunferencia de cintura (CC) fue medida con una cinta métrica no elástica y flexible con una precisión de 0,1 $\mathrm{cm}$, marca Seca. La forma de medición y clasificación de cintura se realizó en base al informe del National Institute of Health del año $1998^{15}$ y del último reporte del año 2014 del MINSAL ${ }^{16}$. La medición de cuatro pliegues cutáneos (tricipital, bicipital, subescapular y suprailíaco) en el lado no dominante del cuerpo fue realizada con un caliper marca Lange de 0,1 milímetro $(\mathrm{mm})$ de precisión, estos valores fueron utilizados para determinar el porcentaje de masa grasa corporal mediante la fórmula de Durnin $1974{ }^{17}$ y su clasificación se realizó según Bray $1998^{18}$.

\section{Hábitos alimentarios}

Con respecto a los hábitos alimentarios, en este estudio se evaluó el cambio en la forma de consumir los alimentos de cada participante antes y al finalizar el periodo de estudio (pre-post intervención) en cuanto a horarios, tiempos de comida y porciones de intercambio. Para fomentar la realización de estos cambios, en cada una de las tres consultas nutricionales, se trabajó en base a la creación de pautas alimentarias personalizadas según las necesidades energéticas y preferencias alimentarias de cada uno de los participantes. Los datos fueron recolectados a través del instrumento encuesta alimentaria de recordatorio de 24 horas (R24h) del día previo a la visita, aplicado en cada consulta. Se evaluó el número de comidas realizadas en el día, el consumo de líquidos totales y de agua, el consumo diario de frutas y de lácteos descremados. El desayuno fue el tiempo de comida que se analizó con mayor profundidad en cuanto a horario, sus componentes, y finalmente el consumo de un desayuno saludable el que debía contener un lácteo descremado, una fruta y un cereal de preferencia integral. Se calculó el Valor Energético Total (VET) para determinar la participación relativa de energía según sustrato, pre y post intervención. EI VET esperado para el desayuno fue de $\mathrm{P} \%=15 \% ; \mathrm{G}=30 \% ; \mathrm{CHO} \%=55 \%$ en base al cual se diseñó la estructura alimentaria de acuerdo a la disponibilidad y preferencias de alimentos de cada sujeto como parte de la intervención. Se escogió este tiempo de comida por ser la comida que realizaron todos los participantes.

\section{Análisis estadístico}

El análisis de datos se realizó con el programa estadístico STATA SE versión 14. Las variables dependientes o "Outcomes" del estudio fueron peso corporal, IMC, perímetro de cintura, porcentaje de grasa corporal y hábitos alimentarios. La distribución normal de variables de tipo continuas fue analizadas con el test de Anderson \& Darling. Entre las medidas descriptivas se trabajó con el promedio y la desviación estándar. Para evaluar el efecto de la intervención sobre la variación del estado nutricional se utilizó la prueba t de Student para muestras pareadas y para evaluar el efecto de la intervención sobre los hábitos alimentarios, las pruebas Chi cuadrado $\left(\mathrm{chi}^{2}\right)$ o Fisher según corresponda. El nivel de significancia fue definido como $\mathrm{P}<0,05$. 


\section{Consideraciones éticas}

Este proyecto fue aprobado por el Comité de Ética de la Facultad de Farmacia, de la Vicerrectoría de Investigación y Desarrollo de la Universidad de Concepción y por el Comité Ético Científico del Servicio de Salud de Concepción. Todos los participantes firmaron el consentimiento informado para participar.

\section{RESULTADOS}

La CNP se aplicó en los tiempos estipulados para cada componente, con un tiempo promedio total de 29,6 $\pm 1,09$ minutos. De los 30 participantes que comenzaron la intervención, solamente 24 completaron los tres meses de intervención ( $20 \%$ de deserción). Las razones de los participantes que abandonaron el estudio fueron debido a motivos personales o traslados fuera de la ciudad.

La anamnesis social arrojó que la edad promedio fue de $41,2 \pm 10,7$ años, siendo un $83 \%$ de los participantes mujeres. Con respecto al estado civil, el $54 \%$ se encontraba casado, el $33 \%$ soltero y $13 \%$ separado. La escolaridad de la muestra fue diversa, el $12 \%$ no finalizó la enseñanza básica o media, 58\% finalizó la enseñanza media, 17\% presentó estudios técnicos superiores y el 13\% universitarios. Finalmente, el $92 \%$ de los participantes se encontraba trabajando al momento de la intervención.

\section{Antropometría}

La Tabla 2 muestra las variables antropométricas estudiadas pre y post intervención. Se puede observar una disminución significativa en todas las variables antropométricas post intervención, siendo el peso corporal $(-2,7 \mathrm{~kg})$ y la circunferencia de cintura $(-2,9 \mathrm{~cm})$ las variables que presentaron mayor reducción. Por otro lado, al estimar el porcentaje de pérdida total con respecto al inicio de la intervención en cada uno de los participantes, se visualiza que en promedio $34 \%$ de los participantes perdieron sobre un $5 \%$ en cualquiera de sus medidas antropométricas iniciales (Tabla 3).

\section{Hábitos alimentarios}

Se determinó la mejoría en relación a los tiempos de comida, siendo la colación de media mañana la única que presentó mejoras significativas $(p<0,001)$. Con respecto al desayuno, existió una mejoría en el número de personas que lo consumían antes de las 9:00 a.m. $(p=0,042)$ y antes de las 9:30 a.m. ( $p=0,003)$. La intervención no logró mejorías significativas sobre un desayuno saludable, sin embargo, si se logró incorporar al menos un lácteo descremado y cereal al desayuno (Tabla 4). Con respecto a la calidad del desayuno, se analizó el valor energético total (VET) de éste en sus diversos componentes. El VET de los participantes (Figura 1) tanto pre como post intervención no fue el adecuado al compararlo con el esperado. El principal componente consumido tanto pre como post intervención, proviene de la energía glucídica. El aporte de energía lipídica fue insuficiente.

Por otro lado, hubo un mayor consumo de una o dos porciones diarias de lácteos descremados $(p<0,001)$. El

\begin{tabular}{|c|c|c|c|c|}
\hline Variables & \multicolumn{3}{|c|}{ Tabla 2} & $\begin{array}{l}\text { Prueba } t \\
\text { (Valor } p \text { ) }\end{array}$ \\
\hline Peso corporal (kg) & $76,3 \pm 13,9$ & $73,6 \pm 14,0$ & $-2,7[-3,52 ;-1,91]$ & $<0,0001^{*}$ \\
\hline Circunferencia de cintura $(\mathrm{cm})$ & $101,6 \pm 9,07$ & $98,7 \pm 8,58$ & $-2,9[-3,91 ;-1,81]$ & $<0,0001^{*}$ \\
\hline
\end{tabular}

Tabla 3

Distribución del porcentaje de personas según pérdida total en cada una de las variables antropométricas.

\begin{tabular}{|c|c|c|c|c|}
\hline Porcentaje de pérdida & $\begin{array}{l}\text { Peso corporal } \\
(\%)\end{array}$ & $\begin{array}{r}\text { IMC } \\
(\%)\end{array}$ & $\begin{array}{c}\text { Porcentaje de grasa } \\
\text { corporal }(\%)\end{array}$ & $\begin{array}{c}\text { Circunferencia de } \\
\text { cintura }(\%)\end{array}$ \\
\hline Sin pérdida & 4 & 4 & 4 & 4 \\
\hline $5-10 \%$ & 25 & 25 & 25 & 17 \\
\hline$\geq 10 \%$ & 4 & 8 & 4 & 0 \\
\hline
\end{tabular}


Tabla 4

Variables alimentarias estudiadas pre y post intervención de los participantes

\begin{tabular}{|c|c|c|c|}
\hline Tiempos de comida & $\%$ & $\%$ & \\
\hline Consumo de colación de media mañana & 42 & 92 & $<0,001^{*}$ \\
\hline Consumo de almuerzo & 100 & 100 & - \\
\hline Consumo de colación de media tarde & 54 & 75 & 0,131 \\
\hline Consumo de colación nocturna & 29 & 21 & 0,740 \\
\hline Desayuno & $\%$ & $\%$ & \\
\hline Consumo antes de las 9:00 AM & 42 & 71 & $0,042 *$ \\
\hline Consumo antes de las 9:30 AM & 50 & 83 & $0,030^{*}$ \\
\hline Consumo desayuno saludable & 8 & 8 & - \\
\hline Una porción & 33 & 92 & $<0,001^{*}$ \\
\hline Dos porciones & 8 & 63 & $<0,001^{*}$ \\
\hline Tres porciones & 0 & 21 & 0,050 \\
\hline Consumo de frutas al día & $\%$ & $\%$ & \\
\hline Una porción & 46 & 83 & $0,015^{*}$ \\
\hline Dos porciones & 29 & 46 & 0,233 \\
\hline Tres porciones & 0 & 12 & 0,234 \\
\hline Consumo de líquidos al día & $\bar{\chi}$ & $\bar{\chi}$ & \\
\hline Líquidos totales (cc) & 1413 & 2204 & $<0,001^{*}$ \\
\hline
\end{tabular}

consumo total de porciones de fruta al día mejoró, sin embargo, las diferencias logran ser significativas sólo en relación al consumo de una porción $(p=0,015)$. El consumo de líquidos totales y agua promedio diario aumentó en 791 cc y 775 cc respectivamente $(p<0,001)$, el 33\% de los participantes logró cumplir con las recomendaciones de las guías alimentarias chilenas de 6 a 8 vasos de agua al día. Todos estos resultados se visualizan en la Tabla 4.

\section{DISCUSIÓN}

En este estudio se realizó un plan piloto de protocolización de consulta nutricional para mejorar y dar cumplimiento a todas las acciones técnicas de la consulta nutricional evaluando su efecto en el estado nutricional y los hábitos alimentarios. El protocolo de 30 minutos produjo mejorías significativas en las variables antropométricas estudiadas, observándose que un $67 \%$ de los participantes lograron reducir su peso corporal en $\geq 5 \%$. Esta reducción es clínicamente relevante, ya que, estudios previos han mostrado que personas que han logrado reducir en un $5 \%$ su peso corporal presentan mejoras significativas sobre factores de riesgo cardiovascular tales como presión arterial, perfil lipídico y glicemia. Estos beneficios se mantienen hasta dos años post-intervención ${ }^{19}$. Considerando el perfil epidemiológico del país, el cual sitúa a Chile entre los top 3 países con mayores prevalencias de factores de riesgo cardiovascular en Latino América ${ }^{20}$, la CNP podría ser una forma efectiva de disminuir la prevalencia de obesidad y, por ende, el riesgo cardiovascular de la población chilena.

En Chile se han realizados diferentes intervenciones nutricionales y multidisciplinares, muchas de ellas con resultados positivos y alentadores ${ }^{21,22}$, sin embargo, estas estrategias no siempre continúan en el tiempo o forman parte de los programas del MINSAL. La consulta nutricional actual es una herramienta básica para los nutricionistas, no obstante, a pesar de su gran uso a nivel nacional, no existe una metodología única de cómo aplicarla a nivel país, lo que no ha permitido estandarizar procedimientos.

Este es el primer trabajo que propone un protocolo de CNP por lo que no es posible comparar los resultados con otras poblaciones y/o intervenciones. Sin embargo, a pesar de ser esta intervención una de las primeras en proponer 


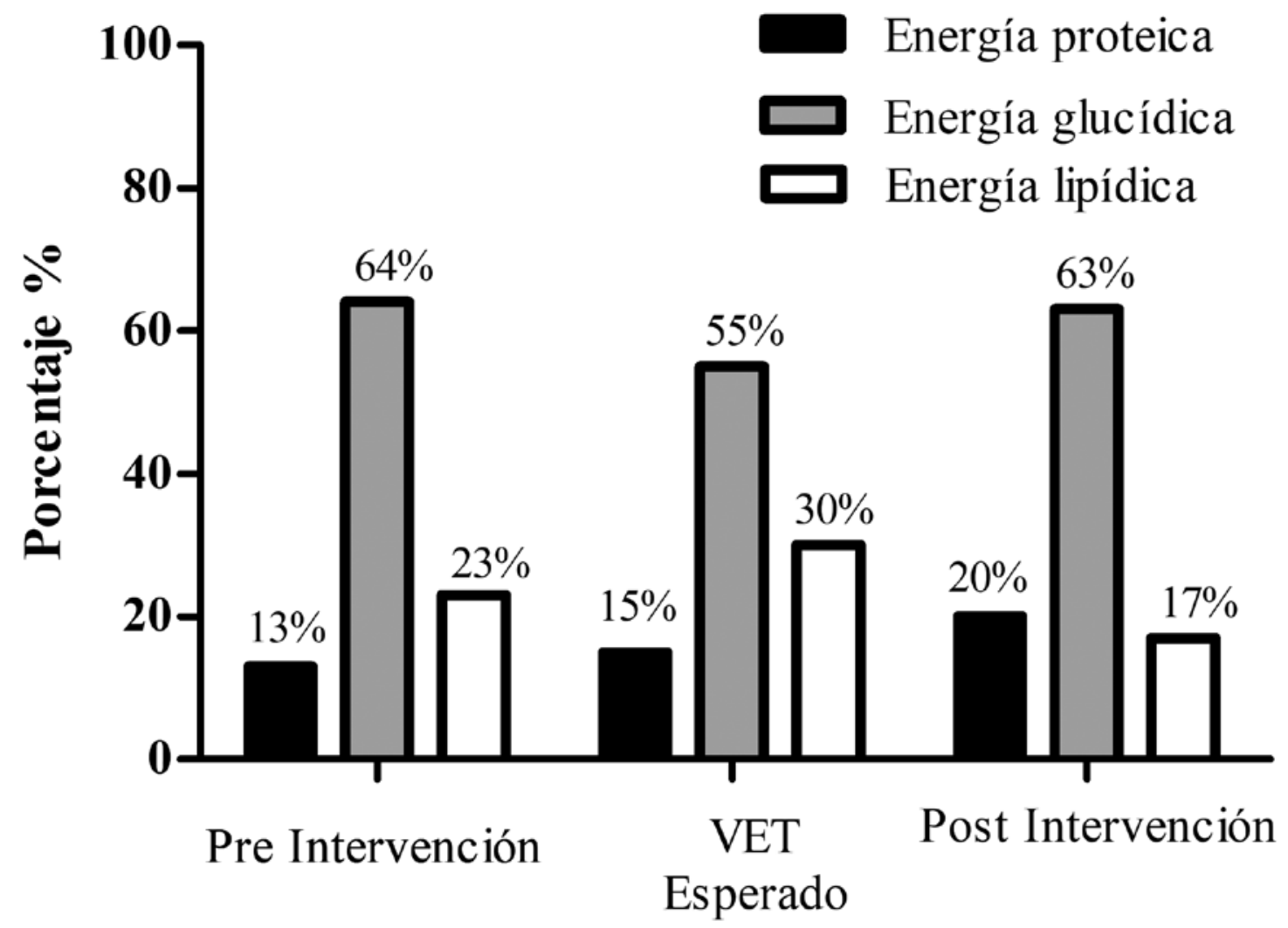

Figura 1. Valor Energético Total (VET) del desayuno pre y post intervención en los participantes

un protocolo nutricional, la protocolización en la práctica médica es un concepto cada vez más recurrente y del cual se han obtenido grandes beneficios. La protocolización permite reducir la variabilidad en la práctica clínica, establecer criterios de atención priorizada y facilitar la recogida de datos, indispensables para la obtención de nuevas evidencias ${ }^{23}$.

Con respecto a los resultados antropométricos, se observaron las mayores variaciones en peso corporal y CC tal y como lo muestra el delta de variación con un valor de $-2,7 \mathrm{~kg}$ y $-2,9 \mathrm{~cm}$ respectivamente. Datos similares se obtuvieron en un estudio realizado por nutricionistas durante un año en Brasil el año 2014, donde igualmente todas las medidas tuvieron una disminución significativa y el delta de variación en un año para peso corporal y CC fue de -2,9 kg y $-3,2 \mathrm{~cm}$, respectivamente ${ }^{10}$.

En relación a los hábitos alimentarios, los datos obtenidos en la Encuesta Nacional de Consumo Alimentario 2014 $(\mathrm{ENCA})^{24}$, señalan que solo un $26,8 \%$ de la población nacional realiza una colación de media mañana. La colación de media mañana ha sido asociada como parte de una alimentación saludable. Un estudio de intervención randomizada reportó que tras 12 meses de intervención mujeres que realizaban una colación a media mañana perdieron un $7 \%$ más de peso corporal en comparación a mujeres que no realizaban esta colación ${ }^{25}$. Tomando en cuenta que nuestro estudio produjo un incremento significativo en el número de personas que adoptaron este hábito alimentario, esto podría explicar en parte la efectividad de la intervención en reducción de marcadores de obesidad. Nuestro estudio reportó que el número de participantes que consumía frutas durante la mañana incrementó en un $46 \%$ post intervención, observándose un incremento principalmente en el grupo que consumió 1 fruta al día. Si bien existe una gran cantidad de evidencia científica en relación al consumo de frutas y su efecto protector contra la obesidad, el incremento del consumo de frutas también ha sido asociado a importantes beneficios cardiovasculares ${ }^{26}$.

No obstante, futuros estudios deberán investigar en mayor detalle qué tipo de frutas aportan mayores beneficios en la reducción de obesidad, ya que se ha reportado que no todas las frutas otorgan los mismos beneficios ${ }^{27}$. Si bien no tomar desayuno se ha asociado a un mayor riesgo de obesidad, la mayor parte de esta evidencia proviene de 
estudios de corte longitudinal. Sin embargo, un reciente estudio de intervención randomizada reportó, que no hay evidencia que sugiera que omitir el desayuno incremente los niveles de obesidad ${ }^{28}$. Si bien nuestro estudio no encontró mejorías en este hábito, esto se debe a que un $92 \%$ de la población intervenida ya reportaba esta conducta antes de la intervención. En cuanto al cumplimiento de un desayuno saludable, se logró la incorporación de cereal y lácteo descremado $(p<0,001)$, y a pesar de que las personas no lograron incorporar fruta durante este tiempo de comida, si lograron aumentar su consumo durante la mañana.

Con relación a la calidad del desayuno no hubo mejorías en la calidad del desayuno medida por VET pre y post intervención. Esto puede deberse a que el alimento sólido fue generalmente pan y porque las preferencias de acompañamiento para el pan, a pesar de las recomendaciones, fueron preparaciones dulces altas en carbohidratos de rápida absorción. En este estudio se logró mejorar el aporte de proteínas a través de la incorporación de lácteos al desayuno, sin embargo, el aporte de grasa disminuyó, probablemente por el temor de los participantes de incorporar frutos secos o palta al ser categorizados como alimentos altos en energía, de alto costo y ajenos a sus hábitos de consumo. Una inadecuada composición nutricional y calidad alimentaria del desayuno podrían relacionarse directamente con falta de energía y rendimiento en las actividades realizadas durante la mañana. En un estudio realizado a adolescentes en México se identificó que a mayor calidad del desayuno era mejor el rendimiento entre los adolescentes ${ }^{29}$.

Las guías alimentarias para la población chilena recomiendan al menos tres porciones de lácteos bajos en grasa al día ${ }^{30}$. Al finalizar la intervención, sólo un $21 \%$ de los participantes logró cumplir con esta recomendación. Los lácteos poseen un papel importante tanto en la capacidad de mantener un peso saludable como en el manejo de la obesidad. Dietas altas en calcio inhiben la lipogénesis, promueven la lipólisis, la oxidación lipídica, la termogénesis e inhiben la obesidad inducida por la dieta en ratones ${ }^{31}$, por lo que potenciar el consumo de dietas altas en calcio podrían generar grandes beneficios en personas con exceso de peso corporal.

Finalmente, con respecto al consumo de líquidos, hubo un aumento de 775 cc de agua en promedio por participante, alcanzando un 33\% de ellos la recomendaciones de 6 a 8 vasos diarios de agua realizada por las guías alimentarias ${ }^{30}$. El consumo de agua reduce agudamente la ingesta de energía de las comidas, por lo que la adopción de este hábito alimentario podría ser uno de los principales factores que favoreció la disminución de peso corporal y otras variables antropométricas. Dennis y cols., evidenciaron que la combinación de una dieta hipocalórica con el consumo de al menos $500 \mathrm{ml}$ de agua antes de cada comida principal conduce a una mayor pérdida de peso corporal que una dieta hipocalórica por si sola en adultos ${ }^{32}$.

Si bien los resultados observados en esta intervención fueron clínicamente relevantes en términos de pérdida de peso corporal, es importante mencionar que este estudio presenta importantes limitaciones que deben ser consideradas al interpretar los resultados. A pesar de ser un estudio de intervención este trabajo carece de un grupo de control y, por ende, no se puede establecer si los cambios observados producto de la intervención serían diferentes a aquellos que tiene acceso a una consulta nutricional no protocolarizada. Por lo tanto, futuras investigaciones deberán confirmar si los efectos de la CNP tienen mejores efectos en la reducción de variables antropométricas y conductas alimentarias que una consulta tradicional. También cabe mencionar que dentro de las fortalezas del estudio se encuentran la medición de variables de interés mediante protocolos estandarizados, y la aplicación de una consulta nutricional que podría ser fácilmente implementada en centros de salud de atención primaria.

\section{CONCLUSIÓN}

La creación de una consulta nutricional protocolizada podría ser una estrategia efectiva para combatir los niveles de obesidad de la población como también así la reducción de factores de riesgo cardiovascular asociados a esta condición. No obstante, es necesario que se corroboren estos beneficios en estudios de mayor duración, con un grupo representativo de participantes de atención primaria y con un grupo control para validar su efectividad, extrapolar sus resultados y corroborar los beneficios obtenidos.

Agradecimientos. Los autores agradecen al Centro de Salud Familiar Chiguayante por facilitar sus dependencias para el desarrollo del estudio y al programa de Magister en Nutrición Humana de la Universidad de Concepción por el financiamiento otorgado.

Conflictos de interés. Los autores declaran no presentar conflictos de interés.

\section{BIBLIOGRAFÍA}

1. Afshin A, Forouzanfar MH, Reitsma MB, Sur P, Estep $K$, Lee $A$, et al. Health Effects of Overweight and Obesity in 195 Countries over 25 Years. NEJM 2017; 377(1): 13-27.

2. WHO. Obesity and overweight. World Health Organization. 2015.

3. MINSAL. National Health Survey 2009-2010. Ministerio de Salud Chile. 2010.

4. M. P. Opinion Study: Users of the Health System Knowledge and Positioning of the Health Superintendence, GES-AUGE and other aspects of the Reform Superintendencia de Salud y Adimark. 2011.

5. MINSAL. Cardiovascular Health Program: Management of the overweight or obese adult. Ministerio de Salud Chile. 2002.

6. MINSAL. Nutritional Intervention Strategy through the Life Cycle for the Prevention of Obesity and Other NonCommunicable Diseases. Ministerio de Salud Chile. 2010.

7. MINSAL. Clinical Guide type 2 Diabetes. Ministerio de Salud Chile. 2010.

8. Dansinger ML, Tatsioni A, Wong JB, Chung M, Balk EM. 
Meta-analysis: the effect of dietary counseling for weight loss. Ann Intern Med 2007; 147(1): 41-50.

9. Soltani S, Shirani F, Chitsazi MJ, Salehi-Abargouei A. The effect of dietary approaches to stop hypertension (DASH) diet on weight and body composition in adults: a systematic review and meta-analysis of randomized controlled clinical trials. Obes Rev 2016; 17(5): 442-454.

10. Ferreira NL, Mingoti SA, Jaime PC, Lopes ACS. Effectiveness of nutritional intervention in overweight women in Primary Health Care. Rev Chil Nutr 2014; 27: 677-687.

11. Tarraga Marcos ML, Rosich N, Panisello Royo JM, Galvez Casas A, Serrano Selva JP, Rodriguez-Montes JA, et al. Efficacy of motivational interventions in the treatment of overweight and obesity. Nutr Hosp 2014; 30(4): 741-748.

12. OMS/OPS. Panorama de la Seguridad Alimentaria y Nutricional en América Latina y el Caribe. Santiago, Chile: Organización de las Naciones Unidas para la Alimentación y la Agricultura, Organización Panamericana de la Salud; 2016 Enero 2017.

13. Outomuro D, Actis AM. Estimation of outpatient consultation time in a medical clinic. Rev Med Chil 2013; 141: 361-366.

14. WHO. Obesity: Preventing and Managing the Global Epidemic. World Health Organization. 2000.

15. NIH. The practical guide to the identification, evaluation and treatment of overweight and obesity in adults. National Institutes of Health 1998.

16. MINSAL. Risk approach for the prevention of cardiovascular diseases. Ministerio de Salud Chile. 2014.

17. Barrera G. Indicators and referents for evaluation of nutritional status, growth and metabolic risk. Instituto de Nutrición y Tecnología de los Alimentos 2008: p 231-237.

18. G. B. What is the ideal body weight? I Nutr Biochem 1998; 9(9): 489-492.

19. Zomer E, Gurusamy K, Leach R, Trimmer C, Lobstein T, Morris $S$, et al. Interventions that cause weight loss and the impact on cardiovascular risk factors: a systematic review and meta-analysis. Obes Rev 2016; 17(10): 1001-1011.

20. Miranda J, Herrera VM, Chirinos JA, Gomez LF, Perel $P$, Pichardo R, et al. Major Cardiovascular Risk Factors in Latin America: A Comparison with the United States. The Latin American Consortium of Studies in Obesity (LASO). Plos One 2013; 8(1): e54056.
21. Henríquez S, Barrera $G$, Hirsch $S$, de la Maza MP, Jara $N$, Leiva $L$, et al. Evaluation of a program for management of metabolic syndrome in adults with overweight and obesity. Rev Med Chil 2014; 142: 817-825.

22. Salinas J, Lera L, González CG, Vio F. Assessment of a nutrition education intervention among construction workers Rev Med Chile 2016; 144: 194-201.

23. Soler Morejón C. Integrated protocols to medical practice future approaches. Rev Habanera Ciencias Med 2011; 10 : 348-354.

24. MINSAL. Final Report National Food Consumption Survey. Ministerio de Salud Chile. 2014.

25. Kong A, Beresford SAA, Alfano CM, Foster-Schubert KE, Neuhouser ML, Johnson DB, et al. Associations between snacking and weight loss and nutrient intake among postmenopausal overweight-to-obese women in a dietary weight loss intervention. J Am Dietetic Assoc 2011; 111(12): 1898-1903.

26. Schwingshackl L, Hoffmann G, Kalle-Uhlmann T, Arregui M, Buijsse B, Boeing H. Fruit and Vegetable Consumption and Changes in Anthropometric Variables in Adult Populations: A Systematic Review and Meta-Analysis of Prospective Cohort Studies. Plos One 2015; 10(10): e0140846

27. Sharma SP, Chung HJ, Kim HJ, Hong ST. Paradoxical Effects of Fruit on Obesity. Nutrients 2016; 8(10). pii: E633.

28. Dhurandhar EJ, Dawson J, Alcorn A, Larsen LH, Thomas EA, Cardel $M$, et al. The effectiveness of breakfast recommendations on weight loss: a randomized controlled trial. Am I Clin Nutr 2014; 100(2): 507-513.

29. Fernández Morales I, Aguilar Vilas MV, Mateos Vega CJ, Martínez Para MC. Relation between the breakfast quality and the academic performance in adolescents of Guadalajara (Castilla-La Mancha). Nutr Hosp 2008; 23: 383-387.

30. MINSAL. Food guides for the Chilean population. Ministerio de Salud Chile. 2013.

31. Zemel MB. The role of dairy foods in weight management. J Am Coll Nutr 2005; 24(6 Suppl): 537S-546S.

32. Dennis EA, Dengo AL, Comber DL, Flack KD, Savla J, Davy $K P$, et al. Water consumption increases weight loss during a hypocaloric diet intervention in middle-aged and older adults. Obesity (Silver Spring, Md) 2010; 18(2): 300-307. 